\title{
RELATIONS BETWEEN STAR FORMATION AND THE INTERSTELLAR MEDIUM
}

\author{
Y. FUKUI AND Y. YONEKURA \\ Department of Astrophysics, Nagoya University
}

\begin{abstract}
We review observational results concerning star formation and dense molecular clouds, the interstellar medium most relevant to starformation process, as well as future prospects.
\end{abstract}

\section{Introduction}

Stars form in interstellar molecular clouds. Recent ideas on star-formation are reviewed in the literature (see e.g., Shu et al. 1987 and references therein). Our knowledge on star formation is obtained mainly by observations of molecular and infrared emission from cold interstellar medium, while the optical data on young stellar objects (YSOs) place important constraints on star-formation theories (e.g., Herbig \& Bell 1988).

The $J=1-0{ }^{12} \mathrm{CO}$ emission at $2.6 \mathrm{~mm}$ was most extensively used to trace molecular clouds in the Galaxy in 1970's and 1980's (see Combes 1991). The most important knowledge obtained through ${ }^{12} \mathrm{CO}$ surveys is that there are 3,000-4,000 giant molecular clouds (GMCs) of $\sim 10^{6} M_{\odot}$ in the Galactic disk, being confined into a spiral arm pattern (Scoville et al. 1987; Dame et al. 1987). These ${ }^{12} \mathrm{CO}$ observations are however either of low resolution and large coverage or of high resolution and small coverage. The goal of next-generation $\mathrm{CO}$ surveys is obviously to cover a larger sky area at resolution high enough to resolve individual sites of star formation in an optically thin probe.

Far-infrared observations made with the IRAS (Infrared Astronomical Satellite) provided a unique tool to probe candidate protostars that emit most of the radiation at far-infrared wavelengths over the whole sky in 1980 's. The relatively high angular resolution, a few arc minutes, was good 
enough to resolve these objects. A protostar (or a protostar candidate, to be exact) is generally invisible, deeply embedded in dense molecular gas, and is observable only at far-infrared and/or sub-millimeter wavelengths. The IRAS catalog provides a comprehensive list of such protostellar objects with a sensitivity limit of $\sim 1 L_{\odot}$ in bolometric luminosity in typical nearby star-forming regions within a few hundred parsecs (Beichman et al. 1986). These objects are perhaps mature protostars close to the end of the mass accretion phase as suggested by their luminosity of $\gtrsim 1-10 L_{\odot}$, high for a low-mass protostar. Next-generation infrared satellites will reveal even earlier stages of a protostar that should appear as a very faint infrared source of $\lesssim 0.1 L_{\odot}$.

Most of the recent observational studies are focused on less than a few tens of well-known regions of star formation. Accordingly, a small fraction of the protostellar IRAS sources have been studied yet, making it uncertain how individual star formation is related to more global properties of star formation. One of such important properties is the stellar initial mass function (IMF), which indicates that most of the stars in the Galaxy are of solar mass, and that the number of stars rapidly decreases with stellar mass (Scalo 1986). We still do not understand the origin of the IMF.

We shall review a recent ${ }^{13} \mathrm{CO}$ survey covering $\sim 17 \%$ of the sky with the Nagoya 4-meter millimeter-wave telescopes. This survey has provided a rich sample of star-forming dense clouds within 1-2 kpc of the Sun. The dataset combined with the IRAS point source catalog indicates that the ${ }^{13} \mathrm{CO}$ clouds better represent star-forming regions on the Galactic scale than ${ }^{12} \mathrm{CO}$. Physical properties of the ${ }^{13} \mathrm{CO}$ clouds are derived by using the sample of nearly 500 clouds, and average characteristics of star formation in the ${ }^{13} \mathrm{CO}$ clouds are summarized.

\section{Star Formation in Giant Molecular Clouds}

We begin with reviewing star formation in GMCs. It is suggested that most of the stars form in GMCs mainly from the fossil records of star formation like OB associations. The number of GMCs we can study in detail is not large; only several GMCs are located within $1 \mathrm{kpc}$ of the Sun. The best studied GMCs are Orion B and Orion A clouds located at $\sim 500 \mathrm{pc}$, for which high-resolution molecular images, and extensive datasets for near and/or far-infrared sources are available.

In the Orion $\mathrm{B}$ cloud five dense CS cores (density $\gtrsim 10^{4} \mathrm{~cm}^{-3}$ ) and three near-infrared clusters are distributed as shown by unbiased surveys made within the spatial extent of the $J=1-0{ }^{12} \mathrm{CO}$ emission (Lada et al. 1991). Three of the CS cores well coincide with the infrared clusters, which 
are interpreted as that star formation occurs almost exclusively as a rich cluster in a dense cloud core.

On the other hand, the dense molecular gas $\left(\mathrm{CS}\right.$ and $\left.\mathrm{C}^{18} \mathrm{O}\right)$ in the Orion A cloud is distributed throughout the cloud more or less continuously, and so is star formation (Fukui et al. 1994; Nagahama et al. 1996). The $J=$ 1-0 $\mathrm{C}^{18} \mathrm{O}$ distribution illustrates that protostellar IRAS sources, molecular outflow sources, and candidate T Tauri stars are all distributed over the cloud with no strong clustering toward individual peaks of dense gas except for the Orion KL region. Infrared imagings indicate that there are several infrared clusters toward luminous protostellar sources (Ori KL, L1641-N, L1641-S, etc., e.g., Fukui et al. 1986; Hodapp 1994), but that they are not so rich in member as in Orion B.

\section{Star Formation in Small Clouds}

The cloud complex in Taurus is located at $\sim 150 \mathrm{pc}$, and is active in lowmass star formation as shown by studies of $\mathrm{T}$ Tauri stars. This complex having a total mass of $\sim 7,000 M_{\odot}$ is not associated with young OB associations nor SNRs, and YSOs are distributed with much lower number density than in Orion clouds, making it one of the best places to test theories on low-mass star formation with no external triggering.

The ${ }^{13} \mathrm{CO}$ distribution of the complex is compared with $\sim 100$ YSOs including T Tauri stars and protostellar IRAS sources (Mizuno et al. 1995). Relatively loose clustering of YSOs toward ${ }^{13} \mathrm{CO}$ peaks indicates that the ${ }^{13} \mathrm{CO}$ clouds are formation sites of these YSOs whose ages are $\lesssim 10^{6}$ yrs. A comparison with the $\mathrm{C}^{18} \mathrm{O}$ distribution on the other hand indicates that protostellar IRAS sources, invisible objects younger than T Tauri stars, are almost always within the $\mathrm{C}^{18} \mathrm{O}$ cores, indicating that the $\mathrm{C}^{18} \mathrm{O}$ cores are the sites of ongoing star formation (Onishi et al. 1996a, 1996b).

The ${ }^{12} \mathrm{CO}$ emission, used to study the Galactic structure or the giant molecular clouds, is no longer a good tracer of individual star formation on a scale of $\sim 1 \mathrm{pc}$. Instead, the ${ }^{13} \mathrm{CO}$ emission that represents density of $\sim$ $10^{3} \mathrm{~cm}^{-3}$ better traces the distribution of YSOs. This is also true for other star-forming clouds of various masses like Orion A, L1251, L1340, IC5146, etc. (e.g., Sato et al. 1994).

\section{A ${ }^{13} \mathrm{CO} J=1-0$ Survey of Star-Forming Clouds}

The preceding discussion suggests that ${ }^{13} \mathrm{CO}$ emission is a good probe of star-forming molecular gas. A ${ }^{13} \mathrm{CO} J=1-0$ survey along the Galactic plane has been carried out by using the two 4-meter millimeter-wave telescopes at Nagoya University since 1990 (e.g., Fukui \& Mizuno 1991; Dobashi et al. 1994, 1996; Yonekura et al. 1996). The observations are 
made at a grid spacing of $8^{\prime}$ in $l$ and $b$ with a $2.7^{\prime}$ beam at $0.1 \mathrm{~km} \mathrm{~s}^{-1}$ velocity resolution. The coverage extends to $\pm 20^{\circ}$ in $b$ over $0^{\circ}-230^{\circ}$ in $l$ corresponding to $\sim 17 \%$ of the sky (Figure 1). 340,000 points have been observed at a $3 \sigma$ noise level equivalent to $1 \times 10^{21} \mathrm{~cm}^{-2}$ in molecular column density. One of the telescopes, named NANTEN telescope, was moved to the Las Campanas Observatory in Chile in 1996 spring in order to extend the survey to the southern sky (Figure 2). Detailed analyses of these ${ }^{13} \mathrm{CO}$ data have been made for regions of Cygnus, Cassiopeia, Perseus, Gemini, and Auriga. The ${ }^{13} \mathrm{CO}$ distribution is highly clumpy and is unambiguously divided into respective ${ }^{13} \mathrm{CO}$ clouds at a $3 \sigma$ noise level. Thus defined ${ }^{13} \mathrm{CO}$ clouds are used to derive physical parameters of the high-density interstellar molecular gas, and to study the mass spectrum, morphology, and dynamical properties of star-forming clouds.

One of the important outcomes of the ${ }^{13} \mathrm{CO}$ survey is that the ${ }^{13} \mathrm{CO}$ clouds are used to identify associated IRAS point sources whose luminosities cannot be otherwise estimated. The association is reliable since most of the ${ }^{13} \mathrm{CO}$ clouds within $1-2 \mathrm{kpc}$ are located at relatively high galactic latitude where confusion with distant galactic sources is negligibly small. Even close to the galactic plane, i.e., at $|b|<1^{\circ}$, more than $70 \%$ of the association is considered to be real according to a statistical test by Yonekura et al. (1996).

Galactic longitude (degree)

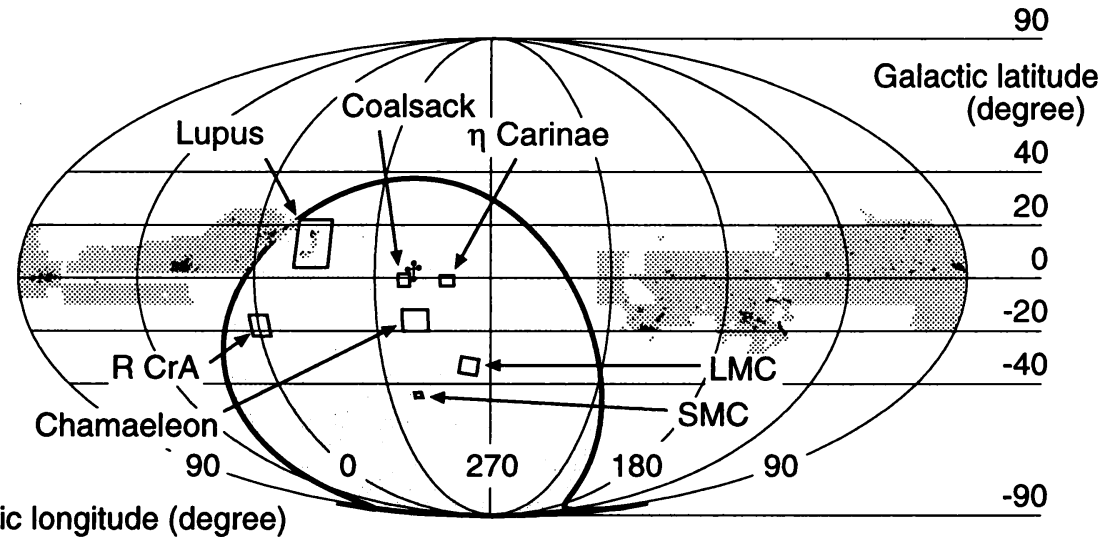

$\square$ Observable from Chile

Already observed at Nagoya

Figure 1. A figure that represents the progress of the Nagoya ${ }^{13} \mathrm{CO}$ survey. The regions where observations have been already finished are represented by hatches. Regions which cannot be observed at Nagoya $\left(\delta<-25^{\circ}\right)$ are represented by brighter hatches. Some well-known star-forming regions in the southern sky are also shown. 


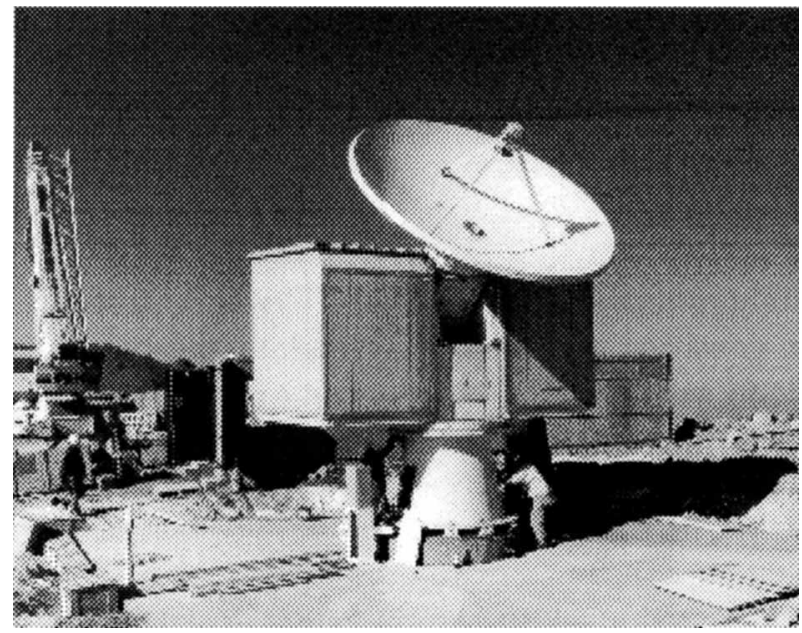

Figure 2. The 4-meter millimeter-wave telescope named NANTEN (=southern sky in Japanese) installed at Las Campanas Observatory in Chile.

\section{5. ${ }^{13} \mathrm{CO}$ Cloud Properties}

In the following we shall present a summary on the cloud properties and star formation in them. We shall confine ourselves to ${ }^{13} \mathrm{CO}$ clouds of $\gtrsim$ $10 M_{\odot}$, and protostellar $I R A S$ sources of $\gtrsim 10 L_{\odot}$, limitations from the detection limits in the ${ }^{13} \mathrm{CO}$ and $I R A S$ surveys, respectively. The number of the cloud analyzed here is 478 and that of the associated IRAS sources is 471 .

(1) Cloud mass spectrum: The mass spectrum of the ${ }^{13} \mathrm{CO}$ clouds is well represented by a power law of an index value of -1.7 ;

$d N_{\text {cloud }}\left(M_{\text {cloud }}\right) / d M_{\text {cloud }} \propto\left(M_{\text {cloud }}\right)^{-1.7}$. A list of the index value for various star-forming regions in the literature indicates that the value is remarkably uniform in a range from -1.3 to -1.8 .

(2) ${ }^{13} \mathrm{CO}$ line width $\Delta V$-size $R$ relation: For the ${ }^{13} \mathrm{CO}$ clouds this relation is very poorly seen as $\Delta V\left({ }^{13} \mathrm{CO}\right) \propto R^{0.18}$ with a correlation coefficient of $<0.4$. The power-law index value is smaller than 0.5 , a value often cited in previous works (e.g., Larson 1981).

(3) Virial mass-LTE mass relation: Virial mass of the ${ }^{13} \mathrm{CO}$ clouds is different from LTE mass calculated from the integrated intensity for an assumed excitation temperature of the CO $J=1-0$ transition, $10 \mathrm{~K}$. The relation between the two masses is represented as follows; $M_{\text {virial }} \propto$ $\left(M_{\mathrm{LTE}}\right)^{0.64}$. This implies that virial mass tends to become larger than LTE mass for small clouds. This deviation is significant particularly for clouds of $\lesssim 10^{3} M_{\odot}$, suggesting that small clouds are not gravitationally 
bound; external pressure may confine them, or alternatively, small clouds are short-lived, with a timescale of $R / \Delta V \sim 10^{6} \mathrm{yrs}$.

(4) Luminosity function of protostellar IRAS sources: We identified 471 IRAS sources having FIR color of $\log [F(25 \mu \mathrm{m}) / F(60 \mu \mathrm{m})] \leq 0$ to be associated with the ${ }^{13} \mathrm{CO}$ clouds. All the IRAS sources thus identified show spectral energy distribution typical to protostars or T Tauri stars, and are definitely detected at three IRAS bands centered at 12,25 , and 60 $\mu \mathrm{m}$. The flux densities were used to calculate the bolometric luminosity, $L_{\mathrm{IR}}$, for the cloud's distance. The luminosity function of the $I R A S$ sources is represented as follows; $d n_{\mathrm{IR}}\left(L_{\mathrm{IR}}\right) / d L_{\mathrm{IR}} \propto\left(L_{\mathrm{IR}}\right)^{-1.45}$. The power-law index seems fairly universal among the regions analyzed here. If we assume a mass to luminosity relation $L_{\mathrm{IR}} \propto m^{3.45}$ for main-sequence stars, the luminosity function can be converted as follows; $d n_{\mathrm{IR}}(m) / d m \propto(m)^{-2.55}$. This relation is consistent with the Scalo's IMF, suggesting that the IMF is universal.

(5) Number of IRAS sources vs. cloud mass: The number of associated IRAS sources in a ${ }^{13} \mathrm{CO}$ cloud is related to the LTE mass as follows; $n_{\mathrm{IR}}\left(M_{\mathrm{LTE}}\right) \propto\left(M_{\mathrm{LTE}}\right)^{0.62}$. This relation can be further used to calculate star formation efficiency (SFE) as follows; SFE $=\Sigma m /\left(M_{\mathrm{LTE}}+\Sigma m\right) \propto$ $\left(M_{\mathrm{LTE}}\right)^{-0.25}$. The dependence of SFE on the cloud mass is very weak and may be described with a small negative power index, suggesting that small clouds can be equally important in star formation on the galactic scale as well as massive clouds. We should note that the statistics is still to be improved by including other regions, and that the mass-to-luminosity relation may be uncertain for protostars whose luminosity is not yet well connected to mass.

\section{Future Prospects}

Our observational knowledge on young stellar objects is still quite limited even for the solar neighborhood of $\lesssim 1-2 \mathrm{kpc}$. This warrants further efforts to make surveys for YSOs at various wavelengths. We expect the following surveys will provide important steps forward in this coming several years:

(1) The existing optical surveys for $\mathrm{T}$ Tauri stars are limited to some well-known regions of star formation. X-ray and near-infrared surveys will provide more comprehensive and complete knowledge on how and where the young stellar objects, mainly $\mathrm{T}$ Tauri stars, are distributed. These surveys include 2MASS, DENIS, and next-generation X-ray satellites such as XMM, ABRIXAS, and AXAF in addition to ROSAT and ASCA.

(2) It is becoming more and more convincing that we are beginning to see protostars which are still growing in mass by accumulating material from the interstellar space. Far-infrared satellites are now observing 
relatively mature protostars close to the end of the mass accretion stage as indicated by their high luminosity, $\gtrsim 1-10 L_{\odot}$, as a low-mass protostar. Next-generation IR satellites, including IRIS and SIRTF in addition to ISO, will be sensitive enough to detect even fainter sources at $100 \mu \mathrm{m}-1$ $\mathrm{mm}$ having $\lesssim 0.1 L_{\odot}$ within $1 \mathrm{kpc}$. These results will provide a strong observational basis to understand the whole evolutionary scenario of a protostar from the early stages of a gaseous condensation and of formation of the initial hydrostatic protostellar core.

(3) Ongoing ${ }^{13} \mathrm{CO}$ and $\mathrm{mm} / \mathrm{sub}$-millimeter surveys both for the northern and southern sky will provide a complete sample of dense cloud cores and nearby star-forming regions. The NANTEN telescope at Las Campanas in Chile will be one of the powerful instruments toward this direction.

We are grateful to the organizing committee for the financial support to participate this fruitful symposium. This research was in part financially supported by the Grants-in-Aid for Scientific Research by the Ministry of Education, Science, and Culture (Nos. 05402003, 06452020, and 07640357). Collaborators of the Nagoya ${ }^{13} \mathrm{CO}$ survey are Y. F., Y. Y., H. Ogawa, A. Mizuno, T. Nagahama, T. Onishi, A. Kawamura, A. Obayashi, K. Tachihara, K. C. Xiao, N. Yamaguchi, A. Hara, T. Hayakawa, S. Kato, N. Mizuno, B. G. Kim, J. P. Bernard, and K. Dobashi.

\section{References}

Beichman, C. A., et al. (1986), Astrophys.J., 307, 337

Combes, F. (1991), Ann.Rev.Astron.Astrophys., 29, 195

Dame, T. M., et al. (1987), Astrophys.J., 322, 706

Dobashi, K., Bernard, J. P. and Fukui, Y. (1996), Astrophys.J., 466, 282

Dobashi, K., et al. (1994), Astrophys.J.Suppl., 95, 419

Fukui, Y. and Mizuno, A. (1991), in E. Falgarone, et al. (eds.), "Fragmentation of Molecular Clouds and Star Formation," Dordrecht, Reidel, 275

Fukui, Y., et al. (1994), in T. Montmerle, et al. (eds.), "Cold Universe," Edition Frontieres, Gif sur Yvett, 157

Fukui, Y., et al. (1986), Astrophys.J.Lett., 311, L85

Herbig, G. H. and Bell, K. R. (1988), Lick Obs. Bull., No.1111

Hodapp, K.-W. (1994), Astrophys.J.Suppl., 94, 615

IRAS Point Source Catalog (1988), Joint IRAS Science Working Group

Lada, E. A., Bally, J. and Stark, A. A. (1991), Astrophys.J., 368, 432

Larson, R. B. (1981), Mon.Not.R.astron.Soc., 194, 809

Mizuno, A., et al. (1995), Astrophys.J.Lett., 445, L161

Nagahama, T., et al. (1996), to be submitted to Astrophys.J.

Onishi, T., et al. (1996a), Astrophys.J., 465, 815

Onishi, T., et al. (1996b), Astrophys.J., submitted

Sato, F., et al. (1994), Astrophys.J., 435, 279

Scalo, J. M. (1986), Fundam. Cosmic Phys., 11, 1

Scoville, N. Z., et al. (1987), Astrophys.J.Suppl., 63, 821

Shu, F. H., Adams, F. C. and Lizano, S. (1987), Ann.Rev.Astron.Astrophys., 25, 23

Yonekura, Y., et al. (1996), Astrophys.J.Suppl., submitted 